Ann. Biol. anim. Bioch. Biophys., I970, 10 (3), 43I-44r.

\title{
MISE EN PLACE ET ÉVOLUTION DE L'ÉQUIPEMENT ENZYMATIQUE DU PANCRÉAS EXOCRINE DU JEUNE RAT PENDANT LA PÉRIODE EMBRYONNAIRE, L'ALLAITEMENT ET LE SEVRAGE
}

\author{
T. CORRING et A. AUMAITRE \\ avec la collaboration technique de Anne-Marie Guevaneau et Jany Marguis \\ Station de Recherches sur l'Élevage des Porcs, \\ Centre national de Recherches zootechniques, 78 -Jouy-en-Josas \\ Institut national de la Recherche agronomique
}

\section{RÉSUMÉ}

L'étude de l'apparition et du développement de quatre enzymes pancréatiques (lipase, amylase, trypsine et chymotrypsine) a été entreprise chez le jeune Rat du I $8 \mathrm{e}$ jour de vie fotale à l'âge de 23 jours, en vue de préciser les modalités de la digestion chez le jeune mammifère au cours des premières périodes de sa vie.

Les principaux résultats indiquent une augmentation générale des activités enzymatiques depuis la naissance et surtout importante de 15 à 18 jours d'âge. Par ailleurs, un comportement particulier des précurseurs protéolytiques (trypsinogène et chymotrypsinogène) est mis en évidence : le taux de chymotrypsinogène est plus élevé que celui du trypsinogène, mais les activités enzymatiques correspondantes, après activation de ces précurseurs, présentent deux périodes d'intense augmentation, au moment de la naissance et après i 8 jours d'âge.

Des conclusions relatives à l'adaptation des sécrétions peuvent être déduites; elles soulignent notamment l'existence d'un seuil dans le développement de l'équipement enzymatique, correspondant à un changement de régime du jeune animal.

\section{INTRODUCTION}

On connait depuis longtemps l'importance des sécrétions digestives et notamment des enzymes pancréatiques dans la digestion des aliments chez le Mammifère adulte (Pavlov, I9I3; Grossman et al., I942; Desnueliz et al., I962). Peu d'études par contre, ont été consacrées à ce sujet chez le jeune. La mise en place de l'équipement enzymatique du pancréas exocrine et son évolution jouent un rôle prépondé- 
rant dès le début de la vie de l'animal. C'est en particulier dans la nutrition du jeune au moment du sevrage que se situent des phénomènes d'une grande importance physiologique.

Quelques auteurs tels que DAHLQuist, (I963) ; HAHN et al. (I966) ; Koldovsky, (I966) ont tenté de caractériser l'apparition d'un " pouvoir digestif " de l'intestin du fœtus de Rat ou de l'embryon humain. D'autres ont étudié les enzymes intestinales de l'animal allaité, en fonction de 1'âge (WALKER, I959; KOLDÖSKY, I966). Mais on n'a généralement pas caractérisé le développement des enzymes pancréatiques avec l'âge. Les études effectuées n'ont porté que sur une période limitée ou sur une enzyme particulière, ou bien encore les stades étudiés couvrent une période très large et ne. sont jamais assez rapprochés pour décrire quantitativement ce développement. Certains auteurs tels que KITTs et al. (I956) ; LEwIs et al. (I957) sur le Porcelet; RUTTER et al., (I968) sur le Raton, ont tenté de déterminer les activités de l'amylase et de la lipase. En ce qui concerne les enzymes protéolytiques, seule une activité "protéolytique totale " dans le pancréas du porcelet a été déterminée (LEwis et al., I957; HARTMAN, I96I). Mais, à notre connaissance, la détermination spécifique de l'acivité de la trypsine ou de la chymotrypsine n'a jamais été effectuée, sur aucun mammifère pendant la période d'allaitement et au moment du sevrage. Après la caractérisation des enzymes dans les organes digestifs et dans le pancréas en particulier, le problème se pose de connaître les mécanismes de leur biosynthèse. Les hypothèses classiques du développement des enzymes en fonction de l'âge ou de la composition du régime subsistent, mais aucun des résultats fragmentaires ne permet de répondre actuellement à une telle question, surtout en raison de la faiblesse des techniques utilisées ou du mode d'expression des résultats.

Ainsi deux explications distinctes sont retenues par les auteurs en ce qui concerne l'adaptation des enzymes pancréatiques. L'évolution avec l'âge a été considérée chez de nombreuses espèces. Certains travaux dus à KuLKA et Duksin (I964) soulignent l'importance de la synthèse de l' $\alpha$-amylase chez le jeune Poulet et cela dès la vie embryonnaire. Elle semble caractériser cette espèce qui est apte à digérer dès la naissance un glucide aussi complexe que l'amidon. De même, KaLLMaN et GroBSTEIN (I964), WESSELS et EVANS (I968), à l'aide du microscope électronique, soulignent la mise en place progressive dans la cellule acineuse du pancréas des ultrastructures qui sont chez l'adulte le siège de la synthèse enzymatique. Nous avons tenté d'augmenter le nombre de ces données en déterminant chez l'embryon de Lapin le stade d'apparition de certaines enzymes protéolytiques (Courtor et al., I970), mais la technique utilisée ne permet pas encore de mesure quantitative.

D'autre part l'explication de la synthèse des enzymes pancréatiques et de la stimulation de leur sécrétion par la composition du régime a été largement confirmée depuis Pavlov (I9I3) chez le Chien, par Desnuelis et al. (I962) et Howard et YUDKIn (1963) chez le Rat.

Dans le présent travail, en vue d'essayer d'éclaircir les phénomènes du développement de la biosynthèse de quelques enzymes, nous avons cherché à étudier l'évolution des principales activités enzymatiques du pancréas exocrine du Rat dès la vie embryonnaire et durant les périodes d'allaitement et de sevrage. 


\section{I. - MATÉRIEI FT MÉTHODES}

\section{Animaux}

La production des jeunes a été réalisée au laboratoire à partir de rates de souche Wistar C. F. de façon à connaître exactement la date de saillie et l'âge des embryons. Le nombre d'animaux de chaque portée est égalisé dès la naissance à I 2 . L'étude a été effectuée sur I 7 portées dont 2 à l'état embryonnaire. Les mères reçoivent un aliment standard du commerce ; cet aliment est consommé spontanément par les ratons. On prélève 2 animaux par portée à chacun des stades suivants :

- naissance, 5, Io, I 5, I 8, 20, 2 I et $23^{\mathrm{e}}$ jour pour les animaux sous la mère ;

- les $18^{\mathrm{e}}$ et $20^{\mathrm{e}}$ jours de la vie fœetale ont été testés sur 2 portées complètes.

$\mathrm{Au}$ cours d'essais préalables, nous avons noté que la variation de l'équipement enzymatique du pancréas exocrine était la plus importante entre le $15^{\mathrm{e}}$ et le $23^{\mathrm{e}}$ jour du jeune animal. C'est la raison pour laquelle les intervalles entre les différents stades sont réduits à partir du $\mathbf{I}_{5}^{\mathrm{e}}$ jour. Le sevrage du raton se situe entre le $2 I^{\mathrm{e}}$ et le $23^{\mathrm{e}}$ jour.

\section{Dosages}

Les animaux sont prélevés sous la mère, le matin, et décapités ; Ie pancréas est immédiatement extrait, pesé frais et homogénéisé à l'aide d'un ultra-turrax Staufen type TP I $8 / 2$ dans l'eau distillée glacée. Les pancréas sont groupés par trois à chacun des stades d'abattage, les activités enzymatiques par pancréas étant trop faibles aux premiers stades pour pouvoir être déterminées.

Après homogénéisation des pancréas, il est procédé à la mesure de la teneur du tissu en protéines totales selon la méthode colorimétrique classique de Folis. Les activités enzymatiques de la chymotrypsine et de la trypsine sont déterminées spécifiquement, après activation des proenzymes par la trypsine cristallisée (Sigma $2 \times$ ), à l'aide d'un titrimètre à pH constant (FrGARELLA et al., 1965). Les substrats synthétiques utilisés sont l'acétyl-l-tyrosine, éthyl-ester (ATEE) et le benzoyl-l-arginine-éthyl-ester (BAEE) respectivement pour la chymotrypsine et la trypsine. L'activité est exprimée en $\mu$ moles d'ATEE et de BAEE hydrolysés par minute.

L'activité de la lipase est déterminée à $37^{\circ} \mathrm{C}$ à l'aide d'un titrimètre à $\mathrm{pH}$ constant par la mesure de la vitesse d'hydrolyse d'une huile d'olive standard émulsifiée dans la gomme arabique à io p. Ioo, selon la méthode de Desnuelie et al., (r962). L'activité est exprimée en $\mu$ moles d'acide gras libérées par minute.

L'activité de l' $\alpha$-amylase est mesurée par la détermination des quantités de sucres réducteurs libérés après une hydrolyse de 20 minutes d'un amidon soluble (Prolabo - 2 I I $53-\mathrm{N}^{\circ}$ du lot 69oI7) (nombre d'extrémités réductrices libérées).

Les différents dosages sont effectués immédiatement après homogénéisation des glandes afin d'éviter une clénaturation des protéines avec le temps.

\section{II. - RÉSULTA'TS}

\section{Développement de la glande pancréatique. Teneur en protéines totales du tissu pancréatique}

L'évolution du poids frais total du pancréas ainsi que la quantité de protéines totales $d u$ tissu pancréatique sont représentées au tableau r. On constate une augmentation de ces valeurs avec l'âge et le poids vif de l'animal.

L'évolution du poids du pancréas rapporté au poids vif est intéressante à considérer : on constate (fig. I) que le rapport varie dans de larges mesures et qu'il existe un coefficient d'allométrie négatif jusqu'au $5^{\mathrm{e}}$ jour, puis constamment positif par la suite. En ce qui concerne la teneur en protéines totales exprimée en fonction du 
TABLEAU I

Poids total du tissu et quantité de protéines totales du pancréas

\begin{tabular}{|c|c|c|c|c|}
\hline Age (jours) & $\begin{array}{l}\text { Poids vif moyen (2) } \\
(\mathrm{g})\end{array}$ & $\begin{array}{l}\text { Poids du pancréas frais } \\
\text { (mg) }\end{array}$ & \multicolumn{2}{|c|}{$\begin{array}{l}\text { Quantité de protéines } \\
\qquad(\mathrm{mg})\end{array}$} \\
\hline Embryons $18\left(^{3}\right)$ & $3,3 \pm 1,0$ & $2^{\prime} \pm 1\left(^{1}\right)$ & - & \\
\hline Embryons $20{ }^{(3)}$ & $4,6 \pm 0,6$ & $33 \pm 2$ & - & \\
\hline Naissance & $6,3 \pm 0,9$ & $37 \pm 1$ & $7,1 \pm$ & 0,2 \\
\hline 5 & $10,5 \pm 1,0$ & $40 \pm 2$ & $5,5 \pm$ & 0,3 \\
\hline 10 & $20,1 \pm 1,7$ & $75 \pm 2$ & $7,8 \pm$ & 0,2 \\
\hline 15 & $31,3 \pm 4,0$ & $110 \pm 15$ & $9,7 \pm$ & 1,2 \\
\hline 18 & $40,2 \pm 5,0$ & $114 \pm 11$ & $13,9 \pm$ & 1,3 \\
\hline 30 & $45,1 \pm 3,7$ & $209 \pm 13$ & $26,5 \pm$ & 1,1 \\
\hline 21 & $50,7 \pm 3,5$ & $232 \pm 20$ & $44,0 \pm$ & 4,0 \\
\hline 23 & $56,9 \pm 7,0$ & $323 \pm 18$ & $65,5 \pm$ & 3,2 \\
\hline
\end{tabular}

(1) Écart-type de la moyenne.

(2) Mâles et femelles.

$\left({ }^{3}\right)$ Jours post-coïtum.

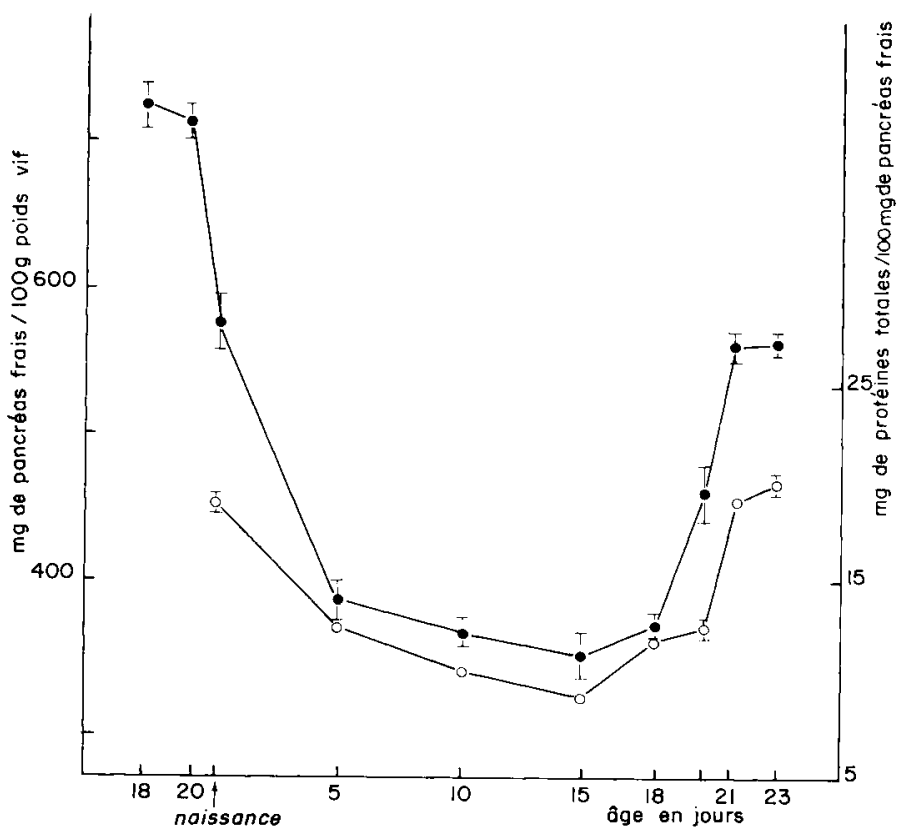

FIG. I. - Variations du poids total du pancréas par $100 \mathrm{~g}$ de poids vif et de la quantité de protéines pancréatiques totales par $100 \mathrm{mg}$ de pancréas frais chez le Rat pendant la période embryonnaive, l'allaitement et le sevrage

- - - mg pancréas frais/roo g poids vif

0 - $\mathrm{mg}$ protéines totales/I00 $\mathrm{mg}$ pancréas frais

Lignes verticales et perpendiculaires (I) : écart-type de la moyenne.

Lorsqu'elles sont inférieures au diamètre des points elles ne sont pas indiquées. 
poids frais de l'organe, on note également que les valeurs enregistrées passent par un minimum au $\mathrm{I}^{\mathbf{e}}$ jour puis augmentent avec l'âge. Il importe de souligner l'étroit parallélisme existant entre le développement pondéral du pancréas par rapport au poids vif et la variation de la teneur en protéines totales du tissu rapportée au poids frais de la glande

\section{Activités enzymatiques}

Les mesures effectuées montrent (tab1. 2) que les diverses enzymes étudiées sont présentes dès le premier stade embryonnaire ( $\mathrm{r} 8^{\mathrm{e}}$ jour de la vie fœetale). Les résultats peuvent être exprimés de deux façons différentes :

a) Activités totales.

Les activités enzymatiques totales, par pancréas, présentent toutes une augmentation avec l'âge de l'animal (tabl. 2, fig. 2). Cependant, des différences apparaissent suivant les enzymes.

TABLEAU 2

Activités enzymatiques totales du pancréas

\begin{tabular}{|c|c|c|c|c|}
\hline $\begin{array}{c}\text { Age } \\
\text { (Jours) }\end{array}$ & $\begin{array}{l}\text { Chymotrypsine } \\
\mu \text { moles ATEE } / \mathrm{mn}\end{array}$ & $\begin{array}{c}\text { Trypsine } \\
\mu \text { moles BAEE } / \mathrm{mn}\end{array}$ & $\begin{array}{c}\text { Lipase } \\
\text { unités }\left({ }^{2}\right)\end{array}$ & $\begin{array}{l}\text { Amylase } \\
\text { unités }\left({ }^{3}\right)\end{array}$ \\
\hline Embryons 18 & $209 \pm 25\left(^{1}\right)$ & $51 \pm 1$ & $55 \pm 12$ & $102 \pm 26$ \\
\hline Embryons 20 & $275 \pm \quad 48$ & $62 \pm 2$ & $85 \pm$ & $195 \mp \quad 3$ \\
\hline Naissance & $709 \pm \quad 26$ & $245 \pm \quad 7$ & $151 \pm$ & $91 \pm$ \\
\hline 5 & $338 \mp 20$ & $132 \pm 6$ & $87 \pm$ & $76 \pm$ \\
\hline 10 & $438 \mp \quad 12$ & $189 \pm 7$ & $1 / 2 \bar{I}$ & $362 \mp \quad 14$ \\
\hline 15 & $526 \pm 73$ & $184 \pm 21$ & $272 \pm \quad 42$ & $599 \pm 70$ \\
\hline 18 & $597 \pm 66$ & $207 \pm 16$ & $556 \pm 49$ & $662 \pm 51$ \\
\hline 20 & $1045 \pm 65$ & $264 \pm 16$ & $252 \pm 108$ & \pm \\
\hline 21 & $1184 \pm 161$ & $338 \pm 21$ & $2544 \pm 174$ & $1532 \pm 166$ \\
\hline 23 & $1787 \pm 51$ & $404 \pm 6$ & $3527 \pm 292$ & $2616 \pm 175$ \\
\hline
\end{tabular}

(1) Écart-type de la moyenne.

( $\left.{ }^{2}\right)$ moles d'acides gras libérées par minute.

( $\left.{ }^{3}\right)$ Nombre d'extrémités réductrices libérées après hydrolyse de 20 minutes d'un amidon soluble (p. de t.).

C'est ainsi que le niveau de la lipase demeure constant jusqu'au $\mathrm{I} 8^{\mathrm{e}}$ jour environ et s'élève rapidement jusqu'au $23^{\mathrm{e}}$ jour, devenant 7 fois plus important durant cette période. Par contre, $1^{\prime} \alpha$-amylase présente une activité dont l'augmentation semble immédiate dès le $5^{\mathrm{e}}$ jour et un léger palier entre le $15^{\mathrm{e}}$ et le $\mathrm{i} 8^{\mathrm{e}}$ jour. Entre le $\mathrm{r} 8^{\mathrm{e}}$ et $23^{\mathrm{e}}$, l'activité augmente environ 3,5 fois. Les activités respectives de la lipase et de $l^{\prime} \alpha$-amylase mesurées à la fin de la vie fœetale et durant les premiers jours qui suivent la naissance sont à peu près identiques.

En ce qui concerne le trypsinogène et le chymotrypsinogène, nous constatons avant et immédiatement après la naissance, un développement différent. En effet, les niveaux des deux précurseurs protéolytiques augmentent brusquement le dernjer 
jour avant la naissance et diminuent sensiblement de la naissance au $5^{\mathrm{e}}$ jour. Ils s'élèvent ensuite régulièrement jusqu'au $\mathrm{I} 8 \mathrm{e}$ jour et de façon plus importante jusqu'au $23^{\mathrm{e}}$ jour.

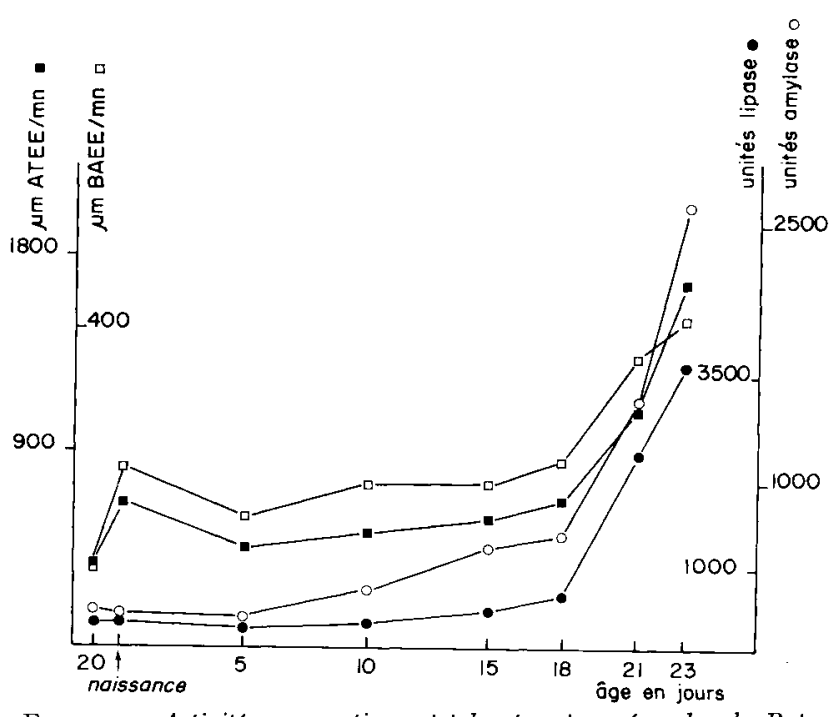

FIG. 2. - Activités enzymatiques totales par pancréas chez le Rat pendant la periode embryonnaire, l'allaitement et le sevrage

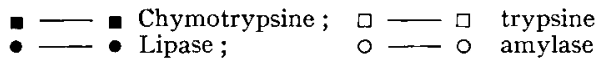

Par ailleurs, entre le $\mathrm{I} 8^{\mathrm{e}}$ et le $23^{\mathrm{e}}$ jour les activités déterminées après activation des précurseurs sont 3 fois et 2 fois plus importantes respectivement pour la chymotrypsine et la trypsine durant cette période.

b) Activités spécifiques.

Lorsque l'on considère les diverses activités exprimées en fonction du poids frais de l'organe (fig. 3), ou par rapport aux protéines totales du tissu, le développement de l'équipement enzymatique du pancréas exocrine présente un comportement particulier. Nous avons considéré le chymotrypsinogène et le trypsinogène (tabl. 3).

L'activité spécifique de la chymotrypsine et de la trypsine, déterminée après activation des précurseurs, est relativement élevée à la naissance, diminue jusqu'au ${ }^{1} 5^{\mathrm{e}}-\mathrm{I} 8 \mathrm{e}$ jour et demeure constante jusqu'au $23^{\mathrm{e}}$ jour. Par ailleurs, l'étude du rapport chymotrypsine/trypsine (tabl. 3) montre que le niveau du chymotrypsinogène est toujours supérieur à celui du trypsinogène. De plus, en fonction de l'âge, on observe que les deux précurseurs se développent de façon parallèle de la naissance au I 8 e jour, puis le niveau relatif du chymotrypsinogène augmente de façon importante du $18^{\mathrm{e}}$ au $23^{\mathrm{e}}$ jour.

La lipase présente aussi une activité spécifique relativement élevée à la naissance ; après une brusque élévation le dernier jour de la vie fotale, cette activitédiminue légèrement jusqu'au $10^{\mathrm{e}}-\mathrm{r} 5^{\mathrm{e}}$ jour puis augmente très rapidement dès $1 \mathrm{e} 15^{\mathrm{e}}$ jour.

Enfin, le taux de l' $\alpha$-am ylase subit une diminution entre le $20^{\mathrm{e}}$ jour de la vie foetale et le $5^{\mathrm{e}}$ jour après la naissance. L'activité de cette enzyme augmente ensuite progressivement et régulièrement jusqu'au $23^{\mathrm{e}}$ jour. 


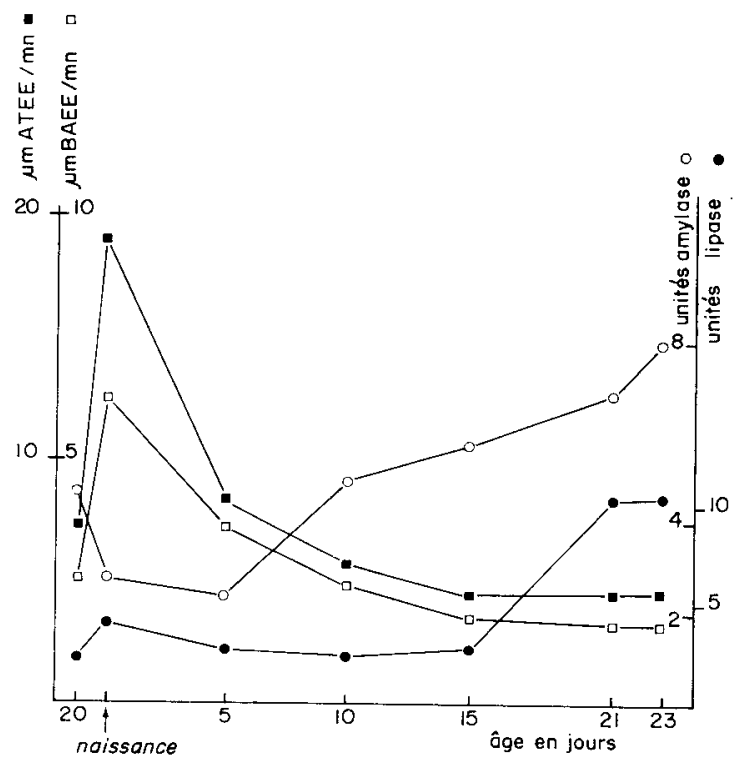

Frg. 3. - Activités enzymatiques par $m g$ de pancréas frais chez le Rat pendant la période embryonnaire, l'allaitement et le sevrage

- - Chymotrypsine; $\square-\square$ Trypsine;

TABLEAU 3

Activités enzymatiques/mg de pancréas frais

\begin{tabular}{|c|c|c|c|c|}
\hline $\begin{array}{l}\text { Age } \\
\text { (jours) }\end{array}$ & $\begin{array}{r}\text { Chymotr } \\
\mu \text { moles } \mathrm{A}\end{array}$ & $\begin{array}{l}\text { psine } \\
\mathrm{EE} / \mathrm{mn}\end{array}$ & $\begin{array}{c}\text { Trypsine } \\
\mu \text { moles BAEE/mn }\end{array}$ & $\frac{\text { Chymotrypsine }}{\text { Trypsine }}$ \\
\hline Embryons 18 & $7,8(e)(2)$ & $\pm 0,09\left({ }^{1}\right)$ & $1,95(e) \pm 0,18$ & 3,9 \\
\hline Embryons 20 & $8,0(d e)$ & $\pm 0,75$ & $1,80(e) \pm 0,18$ & 4,4 \\
\hline Naissance & 19,3 & $\pm 0,45$ & $6,7 \quad \pm 0,30$ & 2,8 \\
\hline 5 & $8, f_{\mathbf{f}}(d)$ & $\pm 0,36$ & $\pm 0,12$ & 2,5 \\
\hline 10 & $5,8(c)$ & $\pm 0,09$ & $\pm 0,06$ & 2,3 \\
\hline 15 & $4,8(b)$ & $\pm 0,06$ & 1,7 (ade) $\pm 0,09$ & 2,7 \\
\hline 18 & $5,2(a)$ & $\pm 0,12$ & $1,8(d e) \pm 0,09$ & 2,9 \\
\hline 20 & $5,0(a b)$ & $\pm 0,09$ & $1,3(b c) \pm 0,06$ & 3,9 \\
\hline 21 & $5,0(a b)$ & $\pm 0,24$ & $1,5(a b) \pm 0,06$ & 3,4 \\
\hline 23 & $5,6(a c)$ & $\pm 0,40$ & $1,3(e) \pm 0,06$ & 4,4 \\
\hline
\end{tabular}

(1) Écart-type de la moyenne.

( $\left.{ }^{\star}\right)$ Les valeurs par colonne affectées d'une même lettre ne sont pas significativement différentes au seuil $\mathrm{P}<0,05$. 


\section{DISCUSSION}

\section{Développement pondéral du pancréas - Protéines totales du tissu}

Nos résultats indiquent que le pancréas du Rat subit un développement pondéral en valeur absolue, en fonction de l'âge, comme chez toutes les espèces étudiées : la Souris (MeUnier et BASTIDE, I968), le Porcelet (KitTs et al., I956, WALKER, I959; HARTMAN et al., I96I), le jeune Enfant (SEIFERT, I959). Il en est de même pour les protéines tissulaires totales dont la quantité globale augmente avec l'âge et le poids vif.

Par ailleurs, lorsque 1'on considère la figure $\mathrm{I}$, les courbes illustrent un développement de la glande pancréatique, moins rapide que celui de l'animal jusqu'aux environs du $5^{\mathrm{e}}$ jour et plus important par la suite (allométrie négative, puis positive). Tout se passe comme si, à partir de cette période, la croissance du pancréas était stimutée.

\section{Activités enzymatiques}

L'examen de nos résultats fait apparaître au moins deux points particuliers :

- L'équipement enzymatique du pancréas exocrine du Rat est en place avant la naissance de 1'animal. Seules des difficultés techniques de prélèvement de la glande ne nous ont pas permis de tester des stades antérieurs au 18 jour de la vie foetale. Par ailleurs, les trop faibles quantités d'organes utilisées pour la détermination des activités de la lipase et de 1 ' $\alpha$-amylase avant la naissance, ne peuvent nous permettre de décrire quantitativement le comportement de ces enzymes. Par contre, grâce à l'utilisation de substrats synthétiques, nous avons pu mesurer la forte élévation du taux de proenzymes protéolytiques, avant le dernier jour de la vie fœtale. Ces faits sont à rapprocher des résultats de KULKA et Duskiv (I964) qui ont observé que 1' $\alpha$ amylase du pancréas de 1'embryon de Poulet augmente considérablement durant la période qui précède l'éclosion de l'œuf. Une variation identique est notée par RUTTER (I965) au bout du $I_{3}$ jour de la vie fœtale de la Souris et à partir du i 8 e jour sur embryons de Rat (Ru'TTER et al., I968).

- Il existe durant la vie du jeune Rat avant le sevrage un seuil dans le développement de l'équipement enzymatique du pancréas exocrine à partir duquel toutes les activités enzymatiques subissent une variation importante. Ce seuil semble se situer à l'âge de I5-I 8 jours et coïncide avec la variation du coefficient d'allométrie observée pour le développement pondéral relatif du pancréas. On constate d'autre part que c'est à partir de ce seuil que toutes les activités enzymatiques totales augmentent considérablement (fig. 2), que les activités trypsique et chymotrypsique exprimées par unité de poids frais demeurent constantes alors qu'elles avaient diminué depuis la naissance (fig. 3) et que le rapport chymotrypsine/trypsine augmente (tab1. 3). Ce seuil à I5-I 8 jours d'âge marquerait un développement intense de la physiologie digestive du pancréas exocrine.

Dans l'ensemble, certains de nos résultats confirment les données partielles obtenues par d'autres auteurs. Ainsi Rokos et al. (I963) observent chez le jeune 
Rat une évolution comparable à celle que nous avons décrite pour la lipase. PRoCHAZKA et al. (I964) montrent également que le taux d' $\alpha$-amylase dans le pancréas augmente rapidement à partir du $\mathrm{I}^{\mathrm{e}}$ jour. Par contre, selon MEUNIER et BASTIDE (I968), la lipase et l'amylase pancréatique de Souris présentent une forte activité à la naissance, qui diminue ensuite, mais à des âges différents pour chaque enzyme. Une variation semblable avec l'âge a été observée pour l' $\alpha$-amylase dans le pancréas du Poulet par Laws et Moore (I963). Sur le Porcelet, KITTs et al., (I956), HuDMAN et al. (I957), WALKER (I959) et HARTMAN et al. (I96I) montrent que l'activité amylasique augmente avec l'âge, tandis que le niveau de la lipase demeure constant. En ce qui concerne les activités de la chymotrypsine et de la trypsine, nos résultats ne peuvent malheureusement pas être comparés.

Il semblerait donc que les principales enzymes du pancréas exocrine soient présentes chez le jeune Mammifère dès la naissance. Durant les premiers jours de la vie, le taux des différentes enzymes étudiées demeurait plus ou moins constant, identique à ce qu'il était le I er jour, ce qui pose le problème de leur rôle dans la digestion du lait. D'après nos résultats, il semblerait que chez le Rat le comportement des précurseurs protéolytiques soit différent des autres protéines enzymatiques et présente deux périodes distinctes d'augmentations spectaculaires au moment de la naissance et à partir du $\mathrm{I} 8 \mathrm{e}$ jour, date à laquelle la nature des protéines du régime varie de façon considérable. Les causes et les mécanismes du développement de cet équipement enzymatique sont actuellement encore totalement inconnus. Un certain nombre d'explications peuvent être présentées.

Parmi celles-ci, nos résultats nous permettent de retenir avec Prochazka et al., (I964) en particulier, que la brusque augmentation du taux de l'amylase au $\mathrm{I} 5^{\mathrm{e}}-\mathrm{I} 8^{\mathrm{e}}$ jour, peut être due à un changement de la nature de la ration alimentaire et à la consommation d'un régime riche en amidon. De plus, l'augmentation du rapport chymotrypsine/trypsine à partir de cette période laisserait supposer une plus grande sensibilité dans la réponse au stimulus alimentaire, de la chymotrypsine, comme l'ont montré DESNUELLE et al. (I962), et notamment une réponse de cette enzyme à un changement dans la nature des protéines du régime (SNOOK et MEYER, I964).

En ce qui concerne la lipase, la réponse de l'activité varie en sens contraire de la proportion de lipides dans le régime. En effet, le lait de la Ratte, très riche en matière grasse, constitue la principale source énergétique du raton allaité. Mais on constate une augmentation de l'activité de la lipase lorsque le taux de lipides de la ration globale du raton diminue dès la consommation d'aliment complémentaire. Ce phénomène démontre une fois de plus (DESNUELLE et al., I962) que la lipase pancréatique ne semble pas subir de variations suivant la composition du régime et notamment sa richesse en graisse. Cependant, et bien que ce travail ne permette aucune vérification, notons que la nature des lipides du lait de la Ratte et de l'aliment solide peut avoir une influence sur l'évolution de la lipase.

Une autre hypothèse pourrait être retenue pour expliquer certaines variations du taux des enzymes avec l'âge. Elle concerne l'effet possible d'une régulation hormonale. Ainsi Prochazka et al., (I964) ont signalé une action des corticoïdes sur la biosynthèse de l'amylase chez le Rat. Ce fait a été confirmé in vitro par Yalovsky et al. (I 969) sur le Poulet. De même, selon BEN ABDELJILL et al. (I965), l'insuline peut avoir un effet particulier sur la biosynthèse de l' $\alpha$-amylase chez le Rat. 


\section{CONCLUSION}

Au cours de cette étude, nous avons pu mettre en évidence certains faits qui semblent mieux caractériser la mise en place et l'évolution de l'équipement enymatique du pancréas exocrine chez le jeune Rat :

I. La glande pancréatique subit un développement pondéral en fonction de l'âge comme c'est le cas chez toutes les espèces étudiées. Le dépôt des protéines tissulaires pancréatiques varie dans le même sens. Cependant ce développement serait stimulé à partir du I $5^{\mathrm{e}}$ jour d'âge.

2. Ires activités enzymatiques déterminées montrent que l'équipement pancréatique exocrine est en place dès la naissance, et se développe régulièrement jusqu'à une période voisine $\mathrm{du} \mathrm{I5}_{5} \mathrm{I} 8^{\mathrm{e}}$ jour à partir de laquelle la quantité totale de ces enzymes augmente de façon importante.

Dans l'ensemble nos résultats montrent que cette période située vers les I5-I 8 e jours d'âge représente dans le développement physiologique du pancréas exocrine un stade très important puisqu'il marquerait le début d'une intense activité de cette glande digestive.

Reçu pour publication en avril 1970.

\section{SUMMARY}

\section{FORMATION AND DEVELOPMENT OF THE ENZYME APPARATUS \\ OF THE EXOCRINE PANCREAS OF YOUNG RATS IN THE EMBRYONIC STAGE AND DURING THE SUCKING AND WEANING PERIODS}

In order to better understand the digestive physiology of the young animal during the sucking period work was undertaken on the appearance and development of the enzyme apparatus of the exocrine pancreas of the young rat while it was being suckled. The following enzymes, chymotrypsin, trypsin, lipase and amylase, were estimated from the I 8 th day of fœtal life until 23 days of age. Activity of the proteolytic enzymes was estimated with specific synthetic substrates.

Total activity of the four enzymes in whole pancreas increased regularly from birth to the I 5 th-I 8 th day and greatly from the I 8 th to the 23 rd day (fig. 2, table 2). Expressed in relation to fresh weight of the organ, lipase and amylase activities increased with age while those of chymotrypsin and trypsin, after falling from birth to $I_{5}$ days remained constant from I $_{5}$ to 23 days of age (fig. 3, table 3). The four enzymes were present from the earliest stage of embryonic life considered.

These results confirm some fragmentary pieces of information obtained with different species of mammal about the development of $\alpha$-amylase in relation to the starch content of the diet. In contrast, development of lipase does not follow changes in the lipid content of the diet.

It seems that the I 5 th-I 8 th days of life constitue a threshold beyond which the exocrine pancreas undergoes intense anatomical and enzymatic development. This development would coincide with the change in diet of the young rat.

The enzyme apparatus of the exocrine pancreas of the young rat is already present at the end of the foetal life and develops regularly with age. At about 15 or 18 days of age it produces a large increase in all the enzyme activities studied. On the other hand, the proteolytic enzymes behave in a particular way, undergoing, in relation to lipase and amylase, development in two well defined stages of life, before birth and after 17 days of age. 


\section{RÉFÉRENCES BIBLIOGRAPHIQUES}

Ben Aboeljill A., Desnuelle P., r964. Sur l'adaptation des enzymes exocrines du pancréas à la composition du régime. Biochim. Biophys. Acta, 81, 136-149.

Ben Abdeljill A., Palla J.-C., Desnuelle P., 1965. Effect of insulin on pancreatic amylase and chymotrypsinogen. Biochim. Biophys. Res. Comm., 18, 7 I-75.

Courtot D., Corring T., Laviolette P., 1970. Détection des proenzymes protéolytiques du pancréas de Lapin par immuno-fluorescence (embryon, nouveau-né, ađulte). Ann. Biol. anim. Bioch. Biophys., 10 (sous presse).

DAHLQUist A., I963. Separation and characterization of two rat intestinal amylases. Biochem. J., $89,272-275$.

Desnuelle P., Reboud J.-P., Ben Abdeljill. A., i962. Influence of the composition of the diet on the enzyme content of rat pancreas in : Ciba Foundation Symposium on the exocrine Pancreas, Churchill (London), p. go.

Figarella C., Taulier J., Sarles H., I965. Dosage de la chymotrypsine et de la trypsine dans le suc duodénal. Bull. Soc. Chim. Biol., 47, 679-686.

Grossmann M. I., Grenngard H., IVy A. C., I942. The effect of dietary composition on pancreatic enzymes. Amer. J. Physiol, 138, 676-682.

Haнn P., Koldovsкy O., I966. Utilization of nutrients during postnatal development. International series of monographs in pure and applied Biology Zoology Division, vol. 33, Pergamon Press.

Hartman P. A., Hays V. W., Baker R. C., Neagle L. H., Catron D. V., ig6r. Digestive.enzyme development in the young pig. J. Anim. Sci., 20, 114-123.

HowARD F., YUDKIN S., 1963. Effect of dietary change upon the amylase and trypsine activities of the rat pancreas. Brit. J. Nutr., 17, 281-295.

Hudman D.S., Friend D. W., Hartman P. A., Ashton G. C., Catron D. V., 1957. Digestive enzyme of the baby pig. Pancreatic and salivary amylase. J. Agric. Food Chim., 5, 69r-693.

Kallman F, Grobstein C., I964. Fine structure of differentiating mouse pancreatic exocrine cell in transfilter culture. J. Cell. Biol., 20, 399-413.

Kitts W. D., Bailey C. B., Woods A. J., I956. The development of the digestive enzyme system of the pig during its preweaning phase of growth. A pancreatic amylase and lipase. Canad.J. Agric. Sci., 36, 46-50.

Koldovsky O., I966. The post-natal development of enzyme activities of the small intestine. In : Development of metabolism as related to nutrition. Karger ed. Basel.

KU1,KA R. G., Dusisin D., I964. Patterns of growth and amylase activity in the developing chick pancreas. Biochim. Biophys. Acta, 91, 506-514.

LAws B. M., MoOrE J. H., 1969. The lipase and esterase activities of the pancreas and small intestine of the chick. Biochem. J., 87, 632-638.

Lewis G. J., Hartman P. A., Lien C. H., Baker R. O., Catron D. V., I957. Digestive enzymes of the baby pig. Pepsin and Trypsin. Agric, Food. Chem., 5, 687.

Meunier M. T., Bastide P., 1968. Variations de quelques activités enzymatiques digestives (lipasique et amylasique) au cours du développement dans le pancréas de Souris. J. Physiol., 60, Suppl, 2, 32-33.

Pavlov I. P., I9r3. The work of the digestive glands. Charles Griffin. ed. London.

Prochazka P., HahN P., Koldovsky O., Noh YNek M., Rokos J., 1964. The activity of $\alpha$-amylase in homogenates of the pancreas of rats during early postnatal development. Physiol. Bohemoslov., 13, 288-29I.

Rokos J., Hahn P., Koldovsky O., Prochazka P., I963. The postnatal development of lipolytic activity in the pancreas and small intestine of the Rat. Physiol. Bohemoslov, 12, 213-218.

Rutter W. J., Wessels N. K., Grobstein C., I965. Control of specific synthetis in the developing pancreas. In : Molecular and cellular aspects of developinent. $38 \mathrm{I}-39 \mathrm{I}$. Ed. Bell E., Harper and Row publishers. N. Y.

Rutter W. J., r968. Regulation of specific protein synthesis in cytodifferenciation. J. Cell Physiol. 72, suppl. I, I-I 8 .

SejFert G., I959. Die exokrine Pankreassekretion in : Die physiologische Entwicklung des Kindes. Linnewey F. 286-289 Springer ed, Berlin.

SNook J. T., Meyer J. H., I964. Responses of digestive enzymes to dietary protein. J. Nutr., 82, 408-4I3.

WALKER D. M., I959. The development of the digestive system of the young animal. II. Carbohydrase enzyme development in the young pig. J. Agric. Sci., 52, 357-37o.

Wessels N. K., Evans J., I968. Ultrastructural studies of early morphogeness is and cytodifferentiation in the embryonic mammalian pancreas. Dev. Biol., 17, 4I3-446.

YALOWSKI U., ZELIKSON R., KULKA G., 1969. The effect of hydrocortisone on the accumulation of amylase in embryonic chick pancreas, Febs Letters, 2, 323-326. 